\section{Guías de Práctica Clínica en el Régimen de Garantías en Salud: una evaluación crítica de su calidad}

\author{
TOMÁS PANTOJA, LORENA VALENZUELA, \\ JAVIERA LÉNIZ, CARLA CASTAÑÓN
}

\section{Clinical practice guidelines in the Chilean health sector reform: a critical assessment of their quality}

Background: Clinical practice guidelines are widely used as tools for improving quality of health care. However, there is increasing concern about limitations in their development process conducting to inconsistent recommendations. During the last decade the use of guidelines has been promoted in the Chilean health system, but their quality has not yet been evaluated systematically. Aim: To assess the quality of clinical practice guidelines developed by the Chilean guidelines program. Material and Methods: All the guidelines developed by the Chilean program between 2005 and 2009 were retrieved from the Ministry of Health website. Each guideline was assessed independently by three appraisers using the Appraisal of Guidelines, Research and Evaluation (AGREE) instrument. Standardized scores were obtained for each dimension in each guideline and across the whole set of guidelines. Results: Sixty guidelines were assessed. The 'scope and purpose' dimension scored significantly higher (mean $82.2 \%$, range: $25.9 \%-100 \%$ ) and the 'applicability' dimension scored significantly lower (mean 23.3\%, range: 0\%-72.4\%) than any other dimension. 'Publication date' was the only variable consistently associated with dimension scores. Conclusions: The quality of Chilean clinical practice guidelines is far from ideal. Although they seem to have a strong sense of purpose and vision, methodological procedures should be strengthened, especially those related to applicability.

(Rev Med Chile 2012; 140: 1391-1400).

Key words: Evaluation studies; Health care reform; Health policy; Health services research; Practice guidelines, as topic.

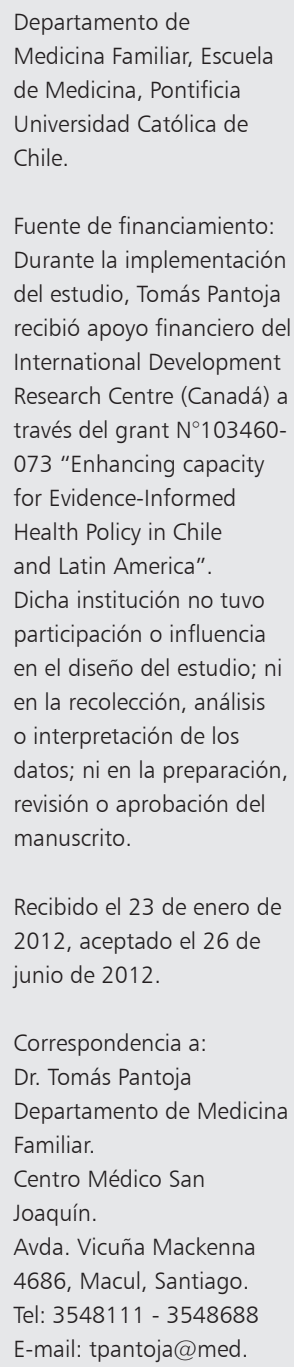

Recibido el 23 de enero de 2012, aceptado el 26 de junio de 2012.

Correspondencia a:

Dr. Tomás Pantoja

Departamento de Medicina

Familiar.

Centro Médico San

Joaquín.

Avda. Vicuña Mackenna

4686, Macul, Santiago.

Tel: 3548111 - 3548688

E-mail: tpantoja@med.

L a calidad del cuidado en salud es un tema relevante para la mayoría de los sistemas de salud en el mundo ${ }^{1-4}$. Chile no ha estado ajeno a dicha tendencia y los temas relacionados con la calidad del cuidado han estado presentes en el proceso de reforma del sector salud, en especial en relación al Régimen de Garantías Explícitas en Salud (RGES) 5 .

Las Guías de Práctica Clínica (GPC) son herramientas utilizadas para mejorar la calidad del cuidado y durante la última década han llegado a ser parte familiar de la práctica clínica en el sistema de salud chileno. Entendidas como 'recomendaciones elaboradas sistemáticamente para asistir a los profesionales de la salud y sus pacientes en la toma de decisiones acerca del cuidado de salud apropiado en condiciones clínicas específicas'6, son promovidas debido a su potencial para mejorar la calidad del cuidado recibido por los pacientes. Sin embargo, pueden verse afectadas por un número de limitaciones ${ }^{7}$. En ese sentido, la diseminación de GPC de dudosa calidad en el sistema de salud puede promover -y eventualmente institucionalizar- el otorgamiento de intervencio- 
nes inefectivas y potencialmente dañinas. Por lo tanto, con el fin de obtener los mejores resultados y limitar los potenciales daños, las GPC deben ser elaboradas usando métodos robustos y deben ser implementadas utilizando estrategias efectivas ${ }^{8,9}$. En relación a su elaboración, una serie de estudios ha revelado importantes deficiencias metodológicas en dominios relacionados con la identificación, evaluación y síntesis de la evidencia científica utilizada en las recomendaciones ${ }^{10}$.

En el sistema sanitario chileno, las GPC han sido elaboradas por un equipo del Ministerio de Salud con diferentes grupos de especialistas y proveedores del sistema de salud público y privado. Aunque se ha promovido su uso, una evaluación formal de su calidad metodológica no ha sido llevada a cabo hasta ahora. Por lo tanto, el objetivo de este estudio fue evaluar la calidad de las GPC elaboradas en el contexto de la implementación del RGES en el sistema de salud chileno. Adicionalmente se intentó identificar aquellos factores asociados con dicha calidad.

\section{Métodos}

\section{Selección de las GPC}

Se incluyeron todas las GPC del RGES elaboradas entre 2005 y 2009. Las guías y los materiales relacionados fueron localizados en el sitio web del Ministerio de Salud. Cuando más de una versión de una GPC estuvo disponible, se utilizó la más reciente.

\section{Evaluación de las GPC}

Aunque existen múltiples instrumentos disponibles para evaluar la calidad de las GPC, aquel elaborado inicialmente por Cluzeau y cols -y posteriormente refinado por el grupo AGREE (Appraisal of Guidelines Research \& Evaluation)es considerado el con mejores propiedades de medición ${ }^{11-14}$. El instrumento AGREE evalúa tanto la calidad del reporte como la de algunos aspectos de las recomendaciones. Se trata de un instrumento que puede ser aplicado a guías de cualquier condición de salud, en sus aspectos preventivos, diagnósticos o terapéuticos. Consta de 23 preguntas con respuestas en una escala ordinal de 1 a 4 y organizadas en seis dominios. Cada dominio intenta evaluar una dimensión separada de la calidad de las guías:
- El alcance y objetivo ('alcance'): el objetivo general de la guía, las preguntas clínicas específicas y la población de pacientes objetivo.

- La participación de los grupos de interés ("participación'): el grado en que la guía representa los puntos de vista de los potenciales usuarios.

- El rigor en la elaboración ('rigor'): el proceso utilizado para reunir y sintetizar la evidencia, los métodos para formular las recomendaciones y actualizar la guía.

- La claridad y presentación ('claridad') se refiere al lenguaje y formato de la guía.

- La aplicabilidad: las implicancias organizacionales, conductuales y financieras de la aplicación de la guía.

- La independencia editorial ('independencia'): la formulación de las recomendaciones no está sesgada por la existencia de conflictos de interés.

Dieciséis profesionales de la salud fueron invitados a participar como evaluadores. Catorce de ellos aceptaron y fueron entrenados en el uso del instrumento AGREE. Hubo 2 sesiones interactivas de capacitación de 4 horas de duración cada una. Cada sesión fue diseñada con una mezcla de presentaciones breves y trabajo en grupo pequeño.

\section{Recolección de la información}

Cada guía seleccionada fue evaluada en forma independiente por tres evaluadores, aplicando el instrumento AGREE. Los evaluadores accedieron a toda la información disponible respecto de cada guía antes de iniciar el proceso de evaluación. Las discrepancias relacionadas con el contenido de las recomendaciones o con el procedimiento de evaluación fueron resueltas a través de discusión abierta y consenso y, en caso de desacuerdo persistente, por un cuarto evaluador ciego a las evaluaciones previas. La información para cada guía y evaluador fue registrada en el formato estandarizado del instrumento AGREE.

\section{Análisis estadístico}

Se obtuvieron los puntajes de cada ítem del instrumento AGREE para cada evaluador. Dada la estructura multidimensional del instrumento, los puntajes fueron calculados independientemente para cada dimensión, sumando los puntajes de los 3 evaluadores para cada uno de los ítems correspondientes a la dimensión y estandarizando 
el total como un porcentaje del máximo puntaje posible. De esta manera los puntajes estandarizados se presentan en un rango entre 0 y $100 \%$. El acuerdo entre evaluadores fue medido utilizando coeficientes de correlación intraconglomerado (ICC) para cada dimensión de cada GPC ${ }^{15,16}$. En el análisis principal, se compararon las medias de los puntajes estandarizados de las seis dimensiones del instrumento AGREE utilizando análisis de varianza. Considerando estudios publicados previamente $^{17,18}$, se evaluó el efecto de las siguientes 4 variables en los puntajes por dimensión, utilizando pruebas univariadas y análisis multivariado: fecha de publicación (2005-2006 versus 2007-2009), participación de las disciplinas relevantes (juzgado por los evaluadores de las GPC como adecuado o no), nivel del sistema de salud (atención primaria versus cuidado hospitalario), ámbito de la GPC (amplio versus restringido). Se consideraron GPC con ámbito amplio a aquellas que incluyeron recomendaciones de tamizaje, diagnóstico y tratamiento, mientras que aquellas de ámbito restringido sólo incluyeron recomendaciones en una de dichas áreas.

El estudio fue aprobado por el Comité de Ética en Investigación de la Escuela de Medicina de la Pontificia Universidad Católica de Chile.

\section{Resultados}

Características de las GPC evaluadas

Se evaluaron 60 GPC para el manejo de 56 condiciones clínicas, incluyendo tamizaje, diagnóstico, prevención y tratamiento. Sin embargo, sólo en 22 de ellas $(36,6 \%)$ se incluyeron todas esas áreas, mientras que en el resto el ámbito fue relativamente más restringido. Veintitrés $(38,3 \%)$ de ellas fueron elaboradas durante 2005-2006, y 37 (61,7\%) durante 2007-2009. La mayoría de ellas $(n=44)$ estaban enfocadas en cuidado hospitalario, con el resto focalizadas en atención primaria. Una lista de las condiciones abordadas por las GPC evaluadas y sus características se presenta en el Anexo 1.

\section{Puntajes estandarizados por dimensión}

La tasa de acuerdo entre los evaluadores fue buena con rangos de ICC entre 0,54 y 0,80 para las diferentes dimensiones. Los puntajes estandarizados por dimensión se presentan en la Figura 1 y la Tabla 1. El puntaje promedio para la dimensión 'alcance' fue de $82,2 \%$ con puntajes sobre $50 \%$ para la mayoría de las GPC $(n=56)$. En el caso de la dimensión 'participación' el puntaje promedio fue $37,5 \%$ con puntajes sobre $50 \%$ para sólo 16 guías.

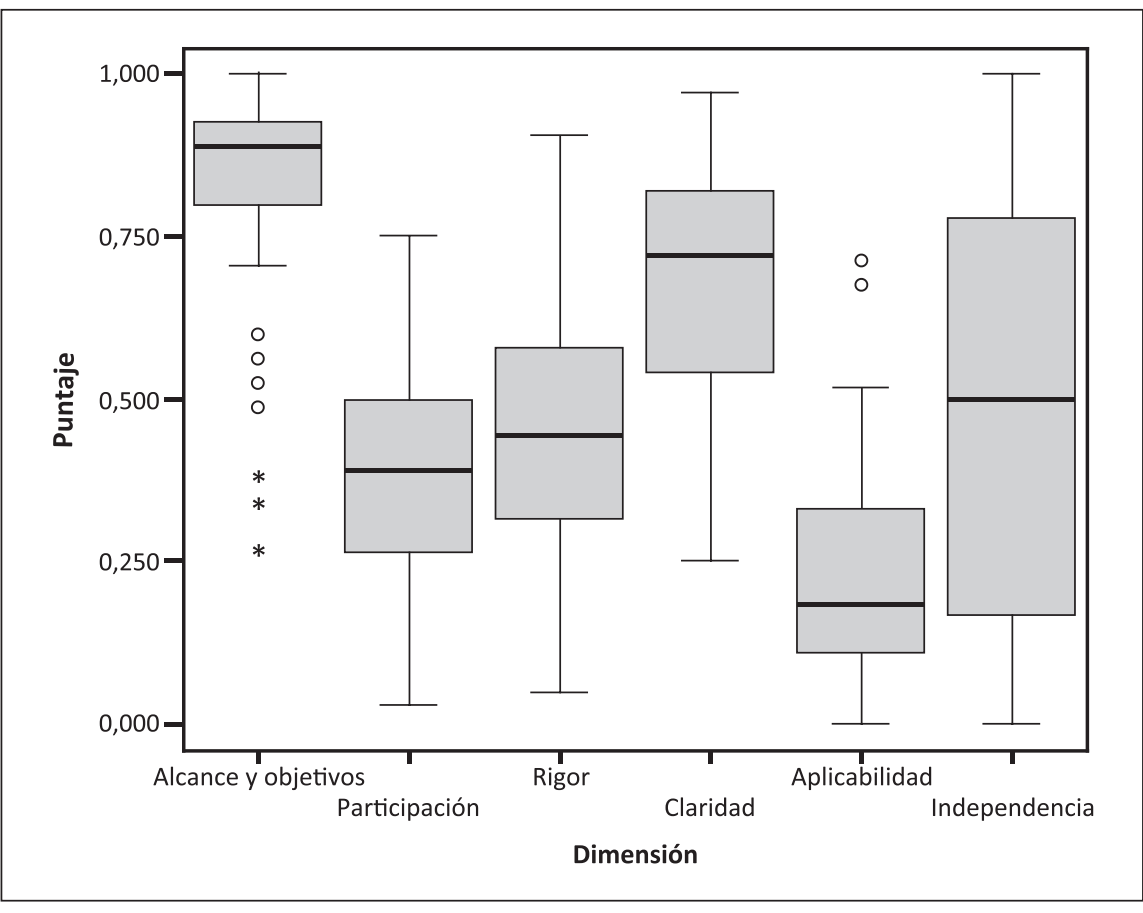

Figura 1. Puntajes estandarizados por dimensión (porcentaje del puntaje máximo posible) para las 60 guías evaluadas. - valores atípicos.

*valores atípicos extremos. 
Evaluación Guías de Práctica Clínica GES 2005-2009 - T. Pantoja et al

Tabla 1. Estadísticos descriptivos para los puntajes estandarizados (porcentaje del puntaje máximo posible) de cada dimensión

\begin{tabular}{|lcccccc|}
\hline Dimensión & Promedio (95\% IC) & Mediana & Mínimo & Máximo & RIQ & CCI (95\% IC) \\
\hline Alcance \& objetivo & $82,2(78,0-86,4)$ & 88,9 & 25,9 & 100,0 & 13,9 & $0,74(0,60-0,84)$ \\
\hline Participación & $37,5(33,7-41,3)$ & 38,9 & 2,8 & 75,0 & 24,3 & $0,74(0,61-0,84)$ \\
\hline Rigor elaboración & $45,7(41,1-50,3)$ & 44,4 & 4,8 & 90,5 & 26,6 & $0,69(0,52-0,80)$ \\
Claridad \& presentación & $68,8(64,3-73,3)$ & 72,2 & 25,0 & 97,2 & 29,1 & $0,54(0,29-0,71)$ \\
Aplicabilidad & $23,3(18,8-27,7)$ & 18,5 & 0,0 & 70,4 & 22,2 & $0,62(0,41-0,76)$ \\
Independencia editorial & $45,3(36,8-53,8)$ & 50,0 & 0,0 & 100,0 & 61,1 & $0,80(0,69-0,87)$ \\
\hline
\end{tabular}

RIQ: rango intercuártil. CCl: coeficiente de correlación intraconglomerado (intracluster correlation coefficient). IC: intervalo de confianza.

En la dimensión 'rigor' el puntaje promedio fue de $45,7 \%$ con 26 guías con un puntaje sobre $50 \%$. Respecto a la 'claridad', el puntaje promedio fue de $68,8 \%$ con 50 de las guías con puntajes por sobre $50 \%$. En la dimensión 'aplicabilidad' el puntaje promedio fue de sólo $23,3 \%$ con la mayoría de las guías $(n=50)$ con puntajes bajo el $50 \%$. Finalmente, el puntaje promedio para 'independencia' fue de $45,3 \%$ con un amplio rango de puntajes (0-100\%), y con la mitad de las guías con puntajes bajo $50 \%$.

\section{Comparación de los puntajes por dimensión}

El análisis de varianza mostró que los puntajes estandarizados para cada dimensión diferían significativamente $(\mathrm{F}=64,105 ; \mathrm{p}<0,001)$. Los análisis post-hoc (procedimiento Tukey HSD) mostraron que: i) el puntaje para la dimensión 'alcance' fue el más alto y que el de 'aplicabilidad' fue el más bajo; ii) el puntaje de 'claridad' fue significativamente más alto que el de 'participación', 'rigor', e 'independencia'; y iii) los puntajes de estas 3 últimas dimensiones no difirieron significativamente.

\section{Variables asociadas con los puntajes por dimensión}

En el análisis univariado las variables 'fecha de publicación' y 'participación de las disciplinas relevantes' estuvieron asociadas a diferencias significativas en los puntajes de cada una de las dimensiones (Tabla 2). Los modelos de regresión múltiple construidos para cada dimensión mostraron que las 4 variables independientes analizadas explicaban entre el 18,3\% ('aplicabilidad') y el $64,2 \%$ ('independencia') de la varianza de los puntajes por dimensión. La variable 'fecha de publicación' dio cuenta de la mayoría de la varianza de los puntajes promedio, mientras que la contribución de las otras variables independientes fue relativamente pequeña o no significativa (Tabla 3 ).

\section{Discusión}

\section{Principales hallazgos}

La calidad metodológica de las GPC implementadas en el RGES está lejos de ser ideal. Aunque las dimensiones 'alcance' y 'claridad' tienen buenos puntajes, existen importantes deficiencias en las otras 4 dimensiones, especialmente en relación a su 'aplicabilidad'. Estos hallazgos son similares a aquellos reportados recientemente por una revisión de 42 estudios (incluyendo un total de 626 GPC) que utilizaron el instrumento AGREE para la evaluación de guías ${ }^{10}$. Dichos autores reportan puntajes por dimensión de $64 \%, 60 \%$, y $22 \%$ para 'alcance', 'claridad' y 'aplicabilidad' respectivamente, y llaman a los elaboradores de GPC a mejorar la calidad de sus productos, proponiendo una serie de acciones para lograrlo ${ }^{10}$.

Un número de factores puede contribuir a esta situación. Primero, los altos puntajes en 'alcance' pudieran estar relacionados a que las guías han sido elaboradas en un programa con un mandato explícito para la producción de estos instrumentos. Segundo, los puntajes intermedios en las dimensiones 'participación' y 'rigor' pudieran deberse a que, aunque existe conocimiento de los conceptos de Medicina Basada en la Evidencia en los grupos elaboradores, no se ha contado con suficientes recursos humanos capacitados para 
Evaluación Guías de Práctica Clínica GES 2005-2009 - T. Pantoja et al

Tabla 2. Puntajes estandarizados por dimensión de acuerdo a características de las guías evaluadas

\begin{tabular}{|c|c|c|c|c|c|c|c|}
\hline \multicolumn{2}{|c|}{ Características de las Guías } & \multicolumn{6}{|c|}{ Puntaje estandarizado por dimensión (promedio) } \\
\hline & & $\begin{array}{c}\text { Alcance \& } \\
\text { objetivo }\end{array}$ & $\begin{array}{l}\text { Partici- } \\
\text { pación }^{2}\end{array}$ & $\begin{array}{c}\text { Rigor } \\
\text { elaboración }\end{array}$ & $\begin{array}{c}\text { Claridad \& } \\
\text { presentación }\end{array}$ & $\begin{array}{l}\text { Aplica- } \\
\text { bilidad }\end{array}$ & $\begin{array}{c}\text { Independencia } \\
\text { editorial }\end{array}$ \\
\hline \multirow[t]{3}{*}{$\begin{array}{l}\text { Fecha } \\
\text { publicación }\end{array}$} & $\begin{array}{l}2005-2006 \\
(n=23)\end{array}$ & 68,6 & 22,8 & 32,1 & 54,7 & 13,5 & 12,6 \\
\hline & $\begin{array}{l}2007-2009 \\
(n=37)\end{array}$ & 90,7 & 46,5 & 54,2 & 77,6 & 29,4 & 65,6 \\
\hline & valor-p $p^{1}$ & $<0,001$ & $<0,001$ & $<0,001$ & $<0,001$ & $<0,001$ & $<0,001$ \\
\hline \multirow{3}{*}{$\begin{array}{l}\text { Participación } \\
\text { disciplinas } \\
\text { relevantes }^{2}\end{array}$} & $\begin{array}{l}\text { Multidisciplinario } \\
(n=46)\end{array}$ & 86,2 & & 49,4 & 72,0 & 27,2 & 54,7 \\
\hline & $\begin{array}{l}\text { Unidisciplinario } \\
(\mathrm{n}=14)\end{array}$ & 69,3 & & 33,8 & 58,1 & 10,6 & 14,3 \\
\hline & valor-p $p^{1}$ & $<0,001$ & & $<0,001$ & 0,023 & $<0,001$ & $<0,001$ \\
\hline \multirow[t]{3}{*}{$\begin{array}{l}\text { Nivel sistema } \\
\text { de salud }\end{array}$} & $\begin{array}{l}\text { Atención primaria } \\
(n=16)\end{array}$ & 73,6 & 39,6 & 49,0 & 70,7 & 25,9 & 42,4 \\
\hline & $\begin{array}{l}\text { Hospital } \\
(n=44)\end{array}$ & 85,4 & 36,7 & 44,5 & 68,1 & 22,4 & 46,4 \\
\hline & valor-p ${ }^{1}$ & 0,078 & 0,592 & 0,478 & 0,643 & 0,610 & 0,701 \\
\hline \multirow[t]{3}{*}{ Ámbito } & $\begin{array}{l}\text { Amplio } \\
(n=22)\end{array}$ & 83,2 & 37,0 & 46,5 & 70,3 & 24,1 & 36,1 \\
\hline & $\begin{array}{l}\text { Restringuido } \\
(n=38)\end{array}$ & 81,7 & 37,7 & 45,3 & 67,9 & 22,9 & 50,6 \\
\hline & valor-p $p^{1}$ & 0,744 & 0,865 & 0,790 & 0,609 & 0,811 & 0,103 \\
\hline
\end{tabular}

'Student's t-test; 'La dimensión 'participación' no fue incluida en esta parte del análisis dado que la variable independiente fue medida utilizando el puntaje obtenido en uno de los ítems de la dimensión (Ítem 4: 'El grupo elaborador de la guía incluyó individuos de todos los grupos profesionales relevantes').

Tabla 3. Porcentaje de la varianza en los puntajes estandarizados por dimensión explicada por las variables independientes seleccionadas utilizando modelos de regresión múltiple

\begin{tabular}{|c|c|c|c|c|c|c|}
\hline \multirow{2}{*}{$\begin{array}{l}\text { Variable independiente } \\
\text { (predictor) }\end{array}$} & \multicolumn{6}{|c|}{ \% de la varianza explicada (R2-ajustada) } \\
\hline & $\begin{array}{c}\text { Alcance \& } \\
\text { objetivo }\end{array}$ & $\begin{array}{l}\text { Partici- } \\
\text { pación }\end{array}$ & $\begin{array}{c}\text { Rigor } \\
\text { elaboración }\end{array}$ & $\begin{array}{c}\text { Claridad \& } \\
\text { presentación }\end{array}$ & $\begin{array}{l}\text { Aplicabi- } \\
\text { lidad }\end{array}$ & $\begin{array}{c}\text { Independencia } \\
\text { editorial }\end{array}$ \\
\hline Fecha de publicación & $41,6^{*}$ & $59,6^{*}$ & $34,3^{*}$ & $39,0^{*}$ & $18,3^{*}$ & $59,3^{*}$ \\
\hline $\begin{array}{l}\text { Participación disciplinas } \\
\text { relevantes }\end{array}$ & ns & NE & ns & ns & ns & $2,6^{* * *}$ \\
\hline Nivel sistema de salud & $9,8^{* *}$ & ns & ns & ns & ns & ns \\
\hline Ámbito & ns & ns & ns & ns & ns & $2,3 * * *$ \\
\hline Todos los predictores & 51,4 & 59,6 & 43,6 & 39,0 & 18,3 & 64,2 \\
\hline
\end{tabular}

${ }^{*} \mathrm{p}<0,001 ;{ }^{* *} \mathrm{p}=0,001 ;{ }^{* *} \mathrm{p}<0,05 ; \mathrm{NE}$ : no evaluado; ns: valor-p no significativo.

implementar un proceso de elaboración de guías con estándares internacionales. En tercer lugar, los bajos puntajes en 'aplicabilidad' pueden estar relacionados a la baja prioridad que este aspecto ha tenido en el RGES, con un proceso más centrado en la entrega de los documentos que en su aplicación en el sistema de salud.
La 'fecha de publicación' fue la variable más consistentemente asociada con la calidad de las guías, con mejores puntajes para aquellas publicadas recientemente comparadas con aquellas elaboradas en los dos primeros años, lo que además se tradujo en una amplia dispersión de los puntajes por dimensión. Esto pudiera reflejar 
un efecto de "curva de aprendizaje" del equipo elaborador, que ha sido capaz de establecer con el tiempo procesos y procedimientos estandarizados para la producción de las guías. Aunque esto es similar a lo ocurrido en España en relación a las guías de AVE isquémico ${ }^{19}$, no ha sido un hallazgo consistente en la literatura ${ }^{18,20-22}$.

\section{Fortalezas y limitaciones de nuestro estudio}

Este es el estudio más reciente en Latinoamérica evaluando la calidad de guías clínicas ${ }^{22-24}$. Representa un avance en relación a nuestro trabajo previo $^{24}$, considerando que el ámbito de aplicación es mayor y que hubo una clara definición de las guías evaluadas. Una segunda fortaleza es el uso de una herramienta validada y estandarizada, que provee un modelo para identificar oportunidades de mejoramiento en el proceso de elaboración de las guías. Sin embargo, se deben mencionar algunas limitaciones de nuestro estudio. La calidad de las guías fue evaluada en base a lo reportado en los documentos disponibles $y$, aunque en algunos casos las guías hacían referencia a documentos adicionales, nuestros intentos por acceder a ellos no fueron exitosos. En segundo lugar, el instrumento AGREE evalúa aspectos metodológicos del proceso de elaboración de las guías y no evalúa la validez clínica de las recomendaciones. Éste es un concepto que ha sido introducido recientemente y cuya definición y métodos de evaluación se encuentran actualmente en desarrollo ${ }^{25}$. Esto permitirá en el futuro realizar juicios respecto tanto a la calidad de las guías como a la validez clínica de sus recomendaciones. Finalmente, justo al momento de finalizar nuestra recolección de información se lanzó una nueva versión del instrumento AGREE ${ }^{26,27}$. Los cambios claves en esta nueva versión fueron una escala de respuesta de 7 puntos (más alineada con los actuales estándares metodológicos de medición), modificaciones menores a la mitad de los ítems, la inclusión de un nuevo ítem relacionado a las fortalezas y limitaciones de la evidencia, y un nuevo Manual del Usuario. Aunque esto representa un avance importante en los métodos de evaluación de la calidad de las guías, el instrumento todavía consta de 23 ítems agrupados en las mismas 6 dimensiones del instrumento original y los cambios a los ítems individuales fueron menores. Por lo tanto, pensamos que nuestros resultados son todavía relevantes y metodológicamente válidos, aún cuándo en el futuro debiéramos utilizar el nuevo instrumento para evaluar las guías que se produzcan en nuestro sistema de salud.

\section{Implicancias para los clínicos y tomadores de decisiones}

Aunque un programa de elaboración de GPC ha sido establecido en el contexto de la implementación del RGES, se debiera trabajar en el mejoramiento de una serie de aspectos metodológicos ${ }^{28-31}$. Dicho trabajo debiera basarse en la experiencia de organizaciones que actualmente elaboran guías en diferentes países ${ }^{32-34}$. Dado los requerimientos de recursos humanos y financieros para la elaboración de GPC de alta calidad, una alternativa es la adaptación de GPC utilizando una aproximación sistemática y válida. Una de dichas aproximaciones ya ha sido utilizada en nuestro país ${ }^{35}$ y podría ser una alternativa para mejorar la eficiencia del proceso de elaboración, especialmente en aquellas áreas donde existe presión política para producir guías en un tiempo reducido. Independientemente de si las GPC son elaboradas 'de novo' o adaptadas, un aspecto que debiera considerarse es la declaración formal de los conflictos de interés por parte de los integrantes del grupo elaborador, dado que la independencia editorial es una dimensión en la que todavía existe espacio de mejoría. Finalmente, para lograr los beneficios deseados en los pacientes, las guías debieran ser implementadas, a través de estrategias que consideren los diferentes aspectos relacionados con su adopción y el diseño de herramientas específicas de apoyo a su implementación local ${ }^{36-39}$.

\section{Preguntas no resueltas e investigación futura}

Aunque ya existe un volumen importante de investigación relacionada con la elaboración e implementación de $\mathrm{GPC}^{8,40}$, dos áreas dónde todavía quedan preguntas por resolver son: la implementabilidad de las guías, y la evaluación de la validez clínica de las recomendaciones. La implementabilidad -definida como 'el set de características que predice la facilidad relativa de implementación de una recomendación' ${ }^{41}$ - es un concepto vinculado a la dimensión 'aplicabilidad' del instrumento AGREE. Aunque ambos están referidos a aspectos intrínsecos de las guías asociadas a su implementación, la aplicabilidad se evalúa a nivel de la guía completa mientras que la implementabilidad se aplicaría a nivel de la recomendación individual. Si bien ya existe 
investigación metodológica en esta área ${ }^{41,42}$, se requiere profundizar en aspectos específicos de su medición, así como en su relación con la calidad metodológica de las guías. El concepto de validez clínica de las recomendaciones está relacionado a las fortalezas y limitaciones de la evidencia que las respalda. Esta área ha sido extensamente trabajada por el grupo GRADE (Grading of Recommendations, Assessment, Development and Evaluation) y dos conceptos centrales son los de la calidad de la evidencia y la fuerza de la recomendación ${ }^{43}$.
Aunque el sistema GRADE es actualmente utilizado por muchas organizaciones elaboradoras de guías, no es el caso de la iniciativa chilena y se requiere mayor trabajo respecto a cuán validas clínicamente son las recomendaciones de las guías evaluadas en este estudio.

Agradecimientos. Especiales agradecimientos a los otros 10 evaluadores (aparte de los autores) que participaron en el estudio, y sin cuyo trabajo este estudio no hubiera sido posible.

Anexo 1. Condiciones clínicas cubiertas por las guías evaluadas y sus características

\begin{tabular}{|c|c|c|c|c|c|}
\hline & Guía & & Caracte & rística & \\
\hline ID & Condición clínica & $\begin{array}{c}\text { Fecha de } \\
\text { publicación }\end{array}$ & $\begin{array}{l}\text { Participación } \\
\text { disciplinas } \\
\text { relevantes } \\
\text { (M: multidisciplinaria; } \\
\text { U: unidisciplinaria) }\end{array}$ & $\begin{array}{c}\text { Nivel sistema } \\
\text { salud } \\
\text { (A: atención primaria; } \\
\text { H: hospital) }\end{array}$ & $\begin{array}{c}\text { Ámbito } \\
\text { (A: amplio; } \\
\text { E: estrecho) }\end{array}$ \\
\hline 1 & Cáncer gástrico & 2009 & M & $\mathrm{H}$ & A \\
\hline 2 & Artrosis leve o moderada de rodilla y/o cadera & 2009 & U & A & A \\
\hline 3 & Cataratas & 2005 & M & $\mathrm{H}$ & $A$ \\
\hline 4 & Vicios de refracción en $>65$ años & 2006 & M & A & A \\
\hline 5 & Infarto agudo del miocardio & 2009 & M & $\mathrm{H}$ & E \\
\hline 6 & Analgesia del parto & 2007 & M & $\mathrm{H}$ & $E$ \\
\hline 7 & Ataque cerebrovascular isquémico & 2007 & M & $\mathrm{H}$ & E \\
\hline 8 & Cáncer de mama & 2006 & M & $\mathrm{H}$ & A \\
\hline 9 & $\begin{array}{l}\text { Endoprótesis total de cadera en > } 65 \text { años con } \\
\text { artrosis de cadera severa }\end{array}$ & 2005 & M & $\mathrm{H}$ & E \\
\hline 10 & Hiperplasia benigna de próstata & 2006 & U & $\mathrm{H}$ & A \\
\hline 11 & Hipoacusia bilateral en > 65 años & 2009 & M & $\mathrm{H}$ & A \\
\hline 12 & Hemorragia subaracnoídea & 2007 & M & $\mathrm{H}$ & $E$ \\
\hline 13 & Hernia del núcleo pulposo lumbar & 2007 & M & $\mathrm{H}$ & E \\
\hline 14 & Linfoma en > 15 años & 2009 & M & $\mathrm{H}$ & $E$ \\
\hline 15 & Gran quemado & 2007 & $M$ & $\mathrm{H}$ & E \\
\hline 16 & Cardiopatías congénitas operables en < 15 años & 2005 & U & $\mathrm{H}$ & A \\
\hline 17 & Displasia broncopulmonar del prematuro & 2009 & M & $\mathrm{H}$ & E \\
\hline 18 & $\begin{array}{l}\text { Síndrome de dificultad respiratoria del recién } \\
\text { nacido }\end{array}$ & 2006 & $U$ & $\mathrm{H}$ & $\mathrm{E}$ \\
\hline 19 & Epilepsia en el niño & 2008 & M & A & E \\
\hline 20 & Estrabismo en $<9$ años & 2006 & U & $\mathrm{H}$ & A \\
\hline 21 & $\begin{array}{l}\text { Hipoacusia neurosensorial bilateral del } \\
\text { prematuro }\end{array}$ & 2009 & M & $\mathrm{H}$ & A \\
\hline 22 & Prevención del parto prematuro & 2005 & M & $\mathrm{H}$ & A \\
\hline 23 & Retinopatía del prematuro & 2009 & M & $\mathrm{H}$ & A \\
\hline 24 & Tumores del sistema nervioso central en adultos & 2007 & M & $\mathrm{H}$ & $\mathrm{E}$ \\
\hline
\end{tabular}




\begin{tabular}{|c|c|c|c|c|c|}
\hline 25 & Esquizofrenia & 2009 & M & $\mathrm{H}$ & A \\
\hline 26 & Insuficiencia renal crónica terminal & 2005 & M & $\mathrm{H}$ & $E$ \\
\hline 27 & Trauma ocular & 2007 & M & $\mathrm{H}$ & $E$ \\
\hline 28 & $\begin{array}{l}\text { Neumonía adquirida en la comunidad } \\
\text { en }>65 \text { años }\end{array}$ & 2005 & $U$ & A & $\mathrm{E}$ \\
\hline 29 & $\begin{array}{l}\text { Alivio del dolor por cáncer avanzado y cuidados } \\
\text { paliativos }\end{array}$ & 2005 & M & $\mathrm{H}$ & $\mathrm{E}$ \\
\hline 30 & Ortesis & 2006 & M & A & $\mathrm{E}$ \\
\hline 31 & $\begin{array}{l}\text { Desprendimiento de retina regmatógeno no } \\
\text { traumático }\end{array}$ & 2006 & $U$ & $\mathrm{H}$ & $E$ \\
\hline 32 & Politraumatizado & 2007 & M & $\mathrm{H}$ & $E$ \\
\hline 33 & Depresión & 2009 & M & A & A \\
\hline 34 & Traumatismo craneoencefálico & 2007 & M & $\mathrm{H}$ & $E$ \\
\hline 35 & Colecitectomía preventiva & 2009 & M & A & A \\
\hline 36 & Asma en $<15$ años & 2006 & $U$ & A & $E$ \\
\hline 37 & Cáncer cervicouterino & 2006 & M & $\mathrm{H}$ & $E$ \\
\hline 38 & Disrafias espinales & 2005 & M & $\mathrm{H}$ & A \\
\hline 39 & Diabetes Mellitus tipo 1 & 2005 & $U$ & $\mathrm{H}$ & $\mathrm{E}$ \\
\hline 40 & Fibrosis quística & 2007 & M & $\mathrm{H}$ & $E$ \\
\hline 41 & Fisura labiopalatina & 2008 & M & $\mathrm{H}$ & $\mathrm{E}$ \\
\hline 42 & Infección respiratoria aguda baja en $<5$ años & 2005 & U & A & $\mathrm{E}$ \\
\hline 43 & Salud oral integral en $<6$ años & 2008 & M & A & A \\
\hline 44 & Escoliosis & 2005 & $U$ & $\mathrm{H}$ & $E$ \\
\hline 45 & Cáncer de testículo en $<15$ años & 2009 & M & $\mathrm{H}$ & $E$ \\
\hline 46 & Salud oral integral en $>60$ años & 2007 & M & A & $\mathrm{E}$ \\
\hline 47 & Hipertensión arterial primaria $>15$ años & 2009 & M & A & $E$ \\
\hline 48 & Urgencia odontológica ambulatoria & 2007 & M & A & $E$ \\
\hline 49 & Diabetes Mellitus tipo 2 & 2009 & M & A & A \\
\hline 50 & $\begin{array}{l}\text { Consumo perjudicial y dependencia de alcohol y } \\
\text { drogas en }<20 \text { años }\end{array}$ & 2007 & M & A & A \\
\hline 51 & Leucemia en $<15$ años & 2008 & M & $\mathrm{H}$ & $E$ \\
\hline 52 & Linfoma en $<15$ años & 2008 & $U$ & $\mathrm{H}$ & $E$ \\
\hline 53 & $\begin{array}{l}\text { Trastornos de generación del impulso cardiaco } \\
\text { que requieren marcapaso }\end{array}$ & 2005 & M & $\mathrm{H}$ & $E$ \\
\hline 54 & Leucemia $>15$ años & 2009 & M & $\mathrm{H}$ & $\mathrm{E}$ \\
\hline 55 & $\mathrm{VIH} / \mathrm{SIDA}$ & 2009 & M & $\mathrm{H}$ & $E$ \\
\hline 56 & Hemofilia & 2009 & M & $\mathrm{H}$ & $E$ \\
\hline 57 & Retinopatía diabética & 2006 & $U$ & $\mathrm{H}$ & A \\
\hline 58 & Artritis reumatoidea & 2007 & $U$ & $\mathrm{H}$ & A \\
\hline 59 & Cáncer de próstata & 2009 & M & $\mathrm{H}$ & A \\
\hline 60 & Enfermedad pulmonar obstructiva crónica & 2006 & M & A & $E$ \\
\hline
\end{tabular}




\section{Referencias}

1. Bodenheimer T. The American Health Care System. The movement for improved quality in health care. N Engl J Med 1999; 340 (6): 488-92.

2. Committe on Quality of Health Care in America IoM. To err is human. Washington: National Academy Press; 2000.

3. Spencer E, Walshe K. Quality and Safety in Healthcare in Europe: A Growing Challenge for Policymakers. Harvard Health Pol Rev 2008; 9 (1): 149-57.

4. Black N. Quality improvement in the NHS. BMJ 2008; 336: 1143.

5. Letelier LM, Bedregal P. Health reform in Chile. Lancet 2006; 368: 2197-8.

6. Institute of Medicine (Field MJ \& Lohr KN eds). Guidelines for Clinical Practice. From Development to Use. Washington DC: National Academy Press; 1992.

7. Woolf SH, Grol R, Hutchinson A, Eccles MP, Grimshaw JM. Potential benefits, limitations, and harms of clinical guidelines. BMJ 1999; 318: 527-30.

8. Oxman AD, Schunemann HJ, Fretheim A, on behalf of the subcommittee on the Use of Research Evidence (SURE) of the WHO Advisory Committee on Health Research (ACHR). Improving the use of research evidence in guidelines development. Oslo: Norwegian Knowledge Centre for the Health Services; 2007.

9. Grimshaw J, Eccles M, Thomas R, MacLennan G, Ramsay C, Fraser C, et al. Toward evidence-based quality improvement. Evidence (and its limitations) of the effectiveness of guideline dissemination and implementation strategies 1966-1998. J Gen Intern Med 2006; 21(Suppl 2): S14-S20.

10. Alonso-Coello P, Irfan A, Sola I, Gich I, Delgado-Noguera $\mathrm{M}$, Rigau $\mathrm{D}$, et al. The quality of clinical practice guidelines over the last two decades: a systematic review of guideline appraisal studies. Qual Saf Health Care 2010; 19 (6): 1-7.

11. Cluzeau FA, Littlejohns P, Grimshaw JM, Feder G, Moran SE. Development and application of a generic methodology to assess the quality of clinical guidelines. Int J Qual Health Care 1999; 11 (1): 21-8.

12. Graham ID, Calder LA, Hébert PC, Carter AO, Tetroe JM. A comparison of clinical practice guideline appraisal instruments. Int JTechnol Assess Health Care 2000; 16 (4): 1024-38.

13. Vlayen J, Aertgeerts B, Hannes K, Sermeus W, Ramaekers D. A systematic review of appraisal tools for clinical practice guidelines: multiple similarities and one common deficit. Int J Qual Health Care 2005; 17 (3): 235-42.

14. The Appraisal of Guidelines Research and Evaluation in Europe (AGREE) Collaborative Group. Development and validation of an international appraisal instrument for assessing the quality of clinical practice guidelines: the AGREE project. Qual Saf Health Care 2003; 12: 18-23.

15. Bravo G, Potvin L. Estimating the reliability of continuous measures with Cronbach's alpha or the intraclass correlation coefficient: toward the integration of two traditions. J Clin Epidemiol 1991; 44 (4-5): 381-90.

16. Prieto L, Lamarca R, Casado A. [Assessment of the reliability of clinical findings: the intraclass correlation coefficient]. Med Clin (Barc) 1998; 110 (4): 142-5.

17. Burgers JS, Grol R, Klazinga NS, Mäkelä M, Zaat J, for the AGREE Collaboration. Towards evidence-based clinical practice: an international survey of 18 clinical guideline programs. Int J Qual Health Care 2003; 15: 31-45.

18. Fervers B, Burgers JS, Haugh MC, Brouwers M, Browman G, Cluzeau FA, Philip T. Predictors of high quality clinical practice guidelines: examples in oncology. Int J Qual Health Care 2005; 17 (2): 123-32.

19. Navarro Puerto MA, Ibarluzea IG, Ruiz OG, Álvarez FM, Herreros RG, Pintiado RE, Domínguez AR, León IM. Analysis of the quality of clinical practice guidelines on established ischemic stroke. Int J Technol Assess Health Care 2008; 24 (3): 333-41.

20. Graham ID, Beardall S, Carter AO, Glennie J, Hebert $\mathrm{PC}$, Tetroe JM, et al. What is the quality of drug therapy clinical practice guidelines in Canada? CMAJ 2001; 165 (2): 157-63.

21. Hurdowar A, Graham ID, Bayley M, Harrison M, WoodDauphinee S, Bhogal S. Quality of stroke rehabilitation clinical practice guidelines. J Eval Clin Pract 2007; 13 (4): 657-64.

22. Esandi ME, Ortiz Z, Chapman E, Dieguez MG, Mejía R, Bernztein R. Production and quality of clinical practice guidelines in Argentina (1994-2004): a cross-sectional study. Implement Sci 2008; 3: 43.

23. Nobre MR, Bernardo WM, Jatene FB. Critical appraisal of 28 guidelines developed by the Brazilian Medical Association. Utveggio Castle, Palermo (Italy) 2003.

24. Pantoja T, Strain H, Valenzuela L. [Clinical practice guidelines in primary health care: A critical appraisal]. Rev Med Chile 2007; 135 (10): 1282-90.

25. Brouwers MC, Kho ME, Browman GP, Burgers JS, Cluzeau F, Feder G, et al. AGREE II: advancing guideline development, reporting and evaluation in health care. J Clin Epidemiol 2010; 63 (12): 1308-11.

26. Brouwers MC, Kho ME, Browman GP, Burgers JS, Cluzeau F, Feder G, et al. Development of the AGREE II, part 1: performance, usefulness and areas for improvement. CMAJ 2010; 182 (10): 1045-52. 
27. Brouwers MC, Kho ME, Browman GP, Burgers JS, Cluzeau F, Feder G, et al. Development of the AGREE II, part 2: assessment of validity of items and tools to support application. CMAJ 2010; 182 (10): E472-8.

28. Fretheim A, Schunemann HJ, Oxman AD. Improving the use of research evidence in guideline development: 3. Group composition and consultation process. Health Res Policy Syst 2006; 4: 15.

29. Fretheim A, Schunemann HJ, Oxman AD. Improving the use of research evidence in guideline development: 15. Disseminating and implementing guidelines. Health Res Policy Syst 2006; 4: 27.

30. Oxman AD, Schunemann HJ, Fretheim A. Improving the use of research evidence in guideline development: 8. Synthesis and presentation of evidence. Health Res Policy Syst 2006; 4: 20.

31. Schunemann HJ, Fretheim A, Oxman AD. Improving the use of research evidence in guideline development: 9. Grading evidence and recommendations. Health Res Policy Syst 2006; 4: 21.

32. Grupo de Trabajo sobre GPC. Elaboración de Guías de Práctica Clínica en el Sistema Nacional de Salud. Manual Metodológico. Madrid (Spain): Plan Nacional para el Sistema Nacional de Salud del Ministerio de Salud y Consumo; 2007.

33. National Institute for Health and Clinical Excellence. The guidelines manual. London: National Institute for Health and Clinical Excellence; 2009.

34. Scottish Intercollegiate Guidelines Network. Sign 50: A guideline developer's handbook. Edinburgh: Scottish Intercollegiate Guidelines Network; 2008.

35. Pantoja T, Ferdinand C, Saldías F, Rojas L, Balcells M, Castro R, et al. Metodología de adaptación de una guía clínica para el manejo de pacientes adultos con neumonia adquirida en la comunidad en una red de salud privada. Rev Med Chile 2011; 139 (11): 1403-13.

36. Grol R, Grimshaw JM. From best evidence to best practice: effective implementation of change in patients' care. Lancet 2003; 362: 1225-30.

37. Grol R, Dalhuijsen J, Thomas S, Veld Ci, Rutten G, Mokkink $\mathrm{H}$. Attributes of clinical guidelines that influence use of guidelines in general practice: observational study. BMJ 1998; 317: 858-61.

38. Michie S, Lester K. Words matter: increasing the implementation of clinical guidelines. Qual Saf Health Care 2005; 14: 367-70.

39. Dijkstra R, Wensing M, Thomas R, Akkermans RP, Braspenning JC, Grimshaw JM, et al. The relationship between organisational characteristics and the effects of clinical guidelines on medical performance in hospitals, a meta-analysis. BMC Health Services Research 2006; 6: 53.

40. Grimshaw JM, Thomas RE, MacLennan G, Fraser C, Ramsay C, Vale L, et al. Effectiveness and efficiency of guideline dissemination and implementation strategies. Health Technol Assess 2004; 8 (6): 1-352.

41. Shiffman RN, Dixon J, Brandt C, Essaihi A, Hsiao A, Michel G, et al. The GuideLine Implementability Appraisal (GLIA): development of an instrument to identify obstacles to guideline implementation. BMC Med Inform Decis Mak 2005; 5: 23.

42. Hill KM, Lalor EE. How useful is an online tool to facilitate guideline implementation? Feasibility study of using eGLIA by stroke clinicians in Australia. Qual Saf Health Care 2009 April; 18 (2): 157-9.

43. Guyatt GH, Oxman AD, Vist GE, Kunz R, Falck-Ytter Y, Alonso-Coello P, et al. GRADE: an emerging consensus on rating quality of evidence and strength of recommendations. BMJ 2008; 336: 924-6. 